\title{
UPPER BOUNDS FOR THE SEPARATION OF REAL ZEROS OF POLYNOMIALS
}

\author{
by PETER WALKER
}

(Received 6th August 1994)

Let $f(x)=\prod_{i}^{n}\left(x-a_{i}\right)$ be a polynomial with distinct real zeros whose separation is defined by $\delta(f)=$ $\min _{i \neq j}\left(a_{i}-a_{j}\right)$. We establish upper estimates for $\delta\left(f^{\prime}-k f\right)$ in terms of $n, k$, and $\delta(f)$. The results give sufficient conditions for the inverse operator $(D-k I)^{-1}$ to preserve the reality of the zeros of a polynomial.

1991 Mathematics subject classification 26 C10.

\section{Introduction}

Let $f$ be a polynomial $f(x)=\prod_{1}^{n}\left(x-a_{i}\right)$ with real zeros which we suppose are in increasing order: $a_{1}<a_{2}<\cdots<a_{n}$. Let $D$ be the differentiation operator, $D f=f^{\prime}$ and let $I$ be the identity.

It is elementary that for real $k$ the property of having only real zeros is preserved by $D-k I$. In [2] we proved more, namely that the separation of the zeros, defined by $\delta(f)=\min _{i}\left(a_{i+1}-a_{i}\right)$, is increased by $D-k I$ (see also [1] for another proof). In [3] we gave an explicit lower bound of the form $\delta\left(f^{\prime}-k f\right) \geqq \delta(f)\left(1+O\left(1 / n k^{2}\right)\right)$.

In this paper we find upper bounds for $\delta\left(f^{\prime}-k f\right)$. The problem is of interest because of its application to the inverse operator $(D-k I)^{-1}$. For $k \neq 0$ the inverse is well-defined on the class of polynomials; explicitly we have for a polynomial of degree $n$ that

and for $k>0$ also

$$
(D-k I)^{-1}=-\frac{1}{k}\left(I+\frac{1}{k} D+\cdots+\frac{1}{k^{n}} D^{n}\right)
$$

$$
(D-k I)^{-1} f(x)=-e^{k x} \int_{x}^{\infty} e^{-k t} f(t) d t
$$

However unlike $D-k I$, the inverse operator does not in general preserve the reality of the zeros. For instance if $f(x)=x^{2}-a^{2}, a>0$, then

$$
(D-k I)^{-1} f(x)=-\left(x^{2}-a^{2}+2 x / k+2 / k^{2}\right) / k
$$

has real zeros only when $|k|>1 / a=2 / \delta(f)$.

In general $(D-k I)^{-1} f$ will have only real zeros when $|k|$ is sufficiently large, and an 
upper bound for $\delta\left(f^{\prime}-k f\right)$ leads to estimates for how large $|k|$ must be. For instance from one of the simpler inequalities of this type, $\delta^{2}\left(f^{\prime}-k f\right) \leqq \delta^{2}(f)+n^{2} / k^{2}$, which was proved in [3], we have equivalently $\delta^{2}\left((D-k I)^{-1} f\right) \geqq \delta^{2}(f)-n^{2} / k^{2}$. Hence if $f$ is a polynomial of degree $n$ with only real zeros then $(D-k I)^{-1} f$ will have only real zeros if $|k|>n / \delta(f)$. The purpose of this paper is to give results of this type which more accurately reflect the dependence on $n$ and $\delta(f)$. For instance Theorem 2 gives $\delta\left(f^{\prime}-k f\right) \leqq \delta(f)+10 h(n) h_{2}(n) /|k|$, where for $x>-1, h(x)=\sum_{k=1}^{\infty}(1 / k-1 /(k+x)$ ) (so that for integer $n, h(n)=1+1 / 2+\cdots+1 / n)$ and $h_{2}(n)=h(h(n))$. It follows that $(D-k I)^{-1}$ preserves the reality of the zeros of $f$ if $|k|>10 h(n) h_{2}(n) / \delta(f)$. The final section gives some calculations which show that these estimates are close to the correct orders of magnitude.

\section{Notation}

As in the introduction, let $f$ be a polynomial $f(x)=\prod_{1}^{n}\left(x-a_{i}\right)$ with real zeros which are in increasing order: $a_{1}<a_{2}<\cdots<a_{n}$. Let $d_{i}=a_{i+1}-a_{i}$ and $d=\delta(f)=\min _{i} d_{i}$. Let $g(x)=f^{\prime}(x) / f(x)=\sum_{1}^{n} /\left(x-a_{i}\right)$.

For $k \in \mathbf{R}$ and $1 \leqq j \leqq n-1$, let $b_{j}$ be the zero of $f^{\prime}-k f$ which lies in the interval $\left(a_{j}, a_{j+1}\right)$; additionally, for $k>0$ let $b_{n}$ be the zero of $f^{\prime}-k f$ with $b_{n}>a_{n}$, while for $k<0$ let $b_{0}$ be the zero of $f^{\prime}-k f$ with $b_{0}<a_{1}$.

Write $b_{j}=a_{j}+u_{j}=a_{j+1}-v_{j+1}$ so that $d_{j}=u_{j}+v_{j+1}$. Let $r_{j}=b_{j+1}-b_{j}=u_{j+1}+v_{j+1}$ so that $\delta\left(f^{\prime}-k f\right)=\min _{j} r_{j}$. Let $\sigma_{j}=\sum_{i \neq j} 1 /\left(a_{j}-a_{i}\right)$.

For $x>-1$ let $h(x)=\sum_{n=1}^{\infty}(1 / n-1 /(x+n))$ from which for $n \in N, h(n)=1+1 / 2+\cdots+$ $1 / n$, and we have the asymptotic expression $h(n)=\log (n)+\gamma+O(1 / n)$ as $n \rightarrow \infty$ and $1 / x>h^{\prime}(x)>1 /(x+1)$ for $x>0$. Let $h_{2}(n)=h(h(n))$ for which $h_{2}(n)=\log \log (n)+\gamma+$ $O(1 / \log n)$ as $n \rightarrow \infty$. It is easy to show inductively that $\sum_{i=1}^{n} 1 /(i h(i)) \leqq 4 h_{2}(n)$.

For $0<x<d$ let $P_{d}(x)=1 / x+1 /(x-d)$ so that $P_{d}$ is a decreasing function from $(0, d)$ onto $\mathbf{R}$. Hence $P_{d}^{-1}$ is a decreasing function from $\mathbf{R}$ onto $(0, d)$ with $P_{d}^{-1}(y)+P_{d}^{-1}(-y)=d$. Explicitly,

$$
P_{d}^{-1}(y)=\frac{2 d}{2+d y+\sqrt{4+d^{2} y^{2}}}
$$

and for $y>0$ we have the estimates $d /(2+d y)<P_{d}^{-1}(y)<1 / y$.

\section{Upper bounds}

We establish estimates for $u_{j}$ and $v_{j}$ in terms of the algebraic function $P_{d}^{-1}$ defined above and use them to deduce results for $r_{j}$.

Lemma 1. For $1 \leqq j \leqq n$ and $k>\sigma_{j}$ we have $u_{j}<1 /\left(k-\sigma_{j}\right)$.

Proof. We know that 


$$
k=g\left(b_{j}\right)=\sum_{i=1}^{N} \frac{1}{a_{j}+u_{j}-a_{i}}=\frac{1}{u_{j}}+\sum_{i \neq j} \frac{1}{a_{j}+u_{j}-a_{i}}
$$

and the terms in the summation are decreasing functions of $u_{j}$, so $k<1 / u_{j}+\sigma_{j}$ and the result follows.

Lemma 2. For all $k \in \mathbf{R}$ and $1 \leqq j \leqq n-1$ we have

and

$$
\left.P_{d_{j}}^{-1}\left(k-\sigma_{j+1}+1 / d_{j}\right)\right) \leqq u_{j} \leqq P_{d_{j}}^{-1}\left(k-\sigma_{j}-1 / d_{j}\right)
$$

$$
v_{j+1} \leqq d_{j}-P_{d_{j}}^{-1}\left(k-\sigma_{j+1}+1 / d_{j}\right) .
$$

Proof. We treat $k$ as a function of $u_{j}$ so $k=\sum_{i=1}^{n} 1 /\left(a_{j}+u_{j}-a_{i}\right)$ and

$$
-\frac{d k}{d u_{j}}=\sum_{i=1}^{n} \frac{1}{\left(u_{j}+a_{j}-a_{i}\right)^{2}}>\frac{1}{u_{j}^{2}}+\frac{1}{\left(u_{j}-d_{j}\right)^{2}} .
$$

Note that as $u_{j} \rightarrow 0_{+}, k \rightarrow \infty$ and $k-1 / u_{j}-\sigma_{j} \rightarrow 0$. Hence we can integrate from 0 to $u_{j}$ to get

$$
\begin{aligned}
& {\left[-k+\frac{1}{u_{j}}\right]_{0}^{u_{j}} \geqq\left[-\frac{1}{u_{j}-d_{j}}\right]_{0}^{u_{j}}} \\
& k-\sigma_{j}-\frac{1}{d_{j}} \leqq \frac{1}{u_{j}}+\frac{1}{u_{j}-d_{j}}=P_{d_{j}}\left(u_{j}\right) \\
& u_{j} \leqq P_{d_{j}}^{-1}\left(k-\sigma_{j}-\frac{1}{d_{j}}\right)
\end{aligned}
$$

as required for one half of the first inequality. Similarly for $v_{j}$ we have $k=$ $\sum_{i=1}^{n} 1 /\left(a_{j}-v_{j}-a_{i}\right)$ and so $d k / d v_{j}>1 / v_{j}^{2}+1 /\left(d_{j-1}-v_{j}\right)^{2}$. If we integrate this from 0 to $v_{j}$ we obtain as above

$$
\begin{aligned}
v_{j} & \leqq P_{d_{j-1}}^{-1}\left(\sigma_{j}-1 / d_{j-1}-k\right) \\
& =d_{j-1}-P_{d_{j-1}}^{-1}\left(k-\sigma_{j}+1 / d_{j-1}\right)
\end{aligned}
$$

which is the required result for $v_{j}$ when we put $j+1$ for $j$. Putting $u_{j}=d_{j}-v_{j+1}$ then gives the other half of the required inequality for $u_{j}$.

Our first theorem gives an estimate of $\delta\left(f^{\prime}-k f\right)$ for large values of $|k|$.

Theorem 1. For all $n \geqq 2$ and $|k| \geqq 2 h(n-1) / \delta(f)$, we have 


$$
\delta\left(f^{\prime}-k f\right) \leqq \delta(f)+\frac{12}{k^{2} \delta(f)}
$$

Proof. From Lemma 1 we have $u_{j}<1 /\left(k-\sigma_{j}\right)$ and from Lemma 2,

$$
\begin{aligned}
v_{j} & <d_{j-1}-P_{d_{j-1}^{-1}}^{-1}\left(k-\sigma_{j}+1 / d_{j-1}\right) \\
& <d_{j-1}-\frac{d_{j-1}}{2+d_{j-1}\left(k-\sigma_{j}+1 / d_{j-1}\right)}
\end{aligned}
$$

using the estimate for $P_{d_{j}}^{-1}$. Hence

$$
\begin{aligned}
u_{j}+v_{j} & <\frac{1}{k-\sigma_{j}}+d_{j-1}-\frac{d_{j-1}}{3+d_{j-1}\left(k-\sigma_{j}\right)} \\
& =d_{j-1}+\frac{3}{\left(k-\sigma_{j}\right)\left(3+d_{j-1}\left(k-\sigma_{j}\right)\right.} \\
& <d_{j-1}+\frac{3}{d_{j-1}\left(k-\sigma_{j}\right)^{2}} .
\end{aligned}
$$

Now choose $j=j_{0}$ say so that $d_{j_{0}-1}=d=\delta(f)$ and

$$
\delta\left(f^{\prime}-k f\right) \leqq u_{j_{0}}+v_{j_{0}}<d+\frac{3}{d\left(k-\sigma_{j_{0}}\right)^{2}}
$$

But $\left|\sigma_{j}\right| \leqq h(n-1) / d$ for all $j$ and so if $k>2 h(n-1) / d \geqq 2\left|\sigma_{j_{0}}\right|$ then $k-\sigma_{j_{0}} \geqq k / 2$ and $\delta\left(f^{\prime}-k f\right) \leqq \delta(f)+12 /\left(\delta(f) k^{2}\right)$ as required. The proof when $k<0$ is similar.

Note that we have proved $\delta\left(f^{\prime}-k f\right) \leqq \delta(f)+O\left(k^{-2}\right)$ as $|k| \rightarrow \infty$ with the implied constant independent of $n$. In the next section we show that the constant can be reduced from 12 to $2+o(1)$ as $k \rightarrow \infty$.

The next theorem gives a result which is valid for all $k \neq 0$.

Theorem 2. For all $n \geqq 2$ and $k \neq 0$,

$$
\delta\left(f^{\prime}-k f\right) \leqq \delta(f)+10 h(n) h_{2}(n) /|k|
$$

Proof. We suppose throught the proof that $j$ is chosen so that $a_{j}=0, a_{j+1}=d=\delta(f)$. We suppose $k>0$; the case $k<0$ is similar.

The proof divides into two cases, determined by the spacing of the points $a_{i}$ with $i<j$. 
When the points are widely separated (which includes the case $j=1$ when there are no points to the left of $a_{j}$ ) we use $r_{j}$ as our upper estimate for $\delta\left(f^{\prime}-k f\right)$. When the separation is less we transfer our attention to the smallest $r_{i}$ with $i<j$.

To be more specific, suppose for the first case that for each $1 \leqq i \leqq j-1$ we have $a_{j-i}<-\operatorname{cih}(i)$ where $c$ is a parameter depending on $n$ and $k$ to be determined later. Then for the second case there is some $1 \leqq i \leqq j-1$ with $a_{j-i} \geqq-\operatorname{cih}(i)$.

In the first case let $F$ be defined by

$$
F(t)=\frac{1}{t}+\sum_{i=0}^{j-1} \frac{1}{t+d+\operatorname{cih}(i)}
$$

and let $u$ be the positive solution of the equation $F(t)=k$. Then $d+u=b_{j+1}$ in the case when all $a_{j-i}=-c i h(i)$ and there are no points with $i>j+1$, and so in general $d+u$ is an upper bound for $b_{j+1}$ and we have $r_{j}<b_{j+1} \leqq d+u$.

We shall show that $u \leqq 2 \operatorname{ch}(n)$ for which, since $F$ is decreasing, it is sufficient to show that $F(2 c h(n)) \leqq k$. But

$$
\begin{aligned}
F(2 c h(n))= & \left.\frac{1}{2 \operatorname{ch}(n)}+\sum_{i=0}^{j-1} \frac{1}{2 \operatorname{ch}(n)+d+\operatorname{cih}(i)}<\frac{1}{c}\left(\frac{1}{2 h(n)}+\sum_{i=0}^{j-1} \frac{1}{2 h(n)+i h(i)}\right)\right) \\
& <\frac{1}{c}\left(\frac{1}{h(n)}+\sum_{i=1}^{j-1} \frac{1}{i h(i)}\right)<\frac{1}{c}\left(\frac{1}{h(n)}+4 h_{2}(j)\right)<\frac{5}{c} h_{2}(n)
\end{aligned}
$$

which equals $k$ if $c=5 h_{2}(n) / k$. Hence in this case $\delta\left(f^{\prime}-k f\right) \leqq d+u \leqq \delta(f)+2 \operatorname{ch}(n) \leqq \delta(f)+$ $10 h(n) h_{2}(n) / k$ as required.

In the second case choose $i \geqq 1$ with $a_{j-1} \geqq-c i h(i)$. Then the sum of the lengths of the $i$ pairs of consecutive intervals $\left(d_{j}+d_{j-1}\right),\left(d_{j-1}+d_{j-2}\right) \ldots\left(d_{j-i+1}+d_{j-i}\right)$ is at most $d+2 \operatorname{cih}(i)$ and so the length of the smallest pair, $d_{s}+d_{s-1}$ say, is at most

$$
(d+2 \operatorname{cih}(i)) / i \leqq d+2 \operatorname{ch}(n) .
$$

Hence since the corresponding interval $\left(b_{s-1}, b_{s}\right)$ of length $r_{s-1}$ is contained in this pair of intervals we have $\delta\left(f^{\prime}-k f\right) \leqq r_{s-1} \leqq \delta(f)+2 c h(n) \leqq \delta(f)+10 h(n) h_{2}(n) / k$ as before, and the proof is complete.

From Theorem 2 we have the following corollary.

Corollary. For any polynomial $f$ of degree $n$ with distinct real zeros, $(D-k I)^{-1} f$ has also distinct real zeros if $|k|>10 h(n) h_{2}(n) / \delta(f)$.

Proof. A continuity argument shows that for sufficiently large $|k|$ the zeros of $(D-k I)^{-1} f$ are close to those of $f$ and hence that all are real when those of $f$ are real. Theorem 2 then gives $\delta\left((D-k I)^{-1} f\right) \geqq \delta(f)-10 h(n) h_{2}(n) /|k|$ for these values of $k$. But the 
zeros depend continuously on $k$ and so $\delta\left((D-k I)^{-1} f\right)$ will remain positive as long as $|k|>10 h(n) h_{2}(n) / \delta(f)$ and so the zeros remain real for these values of $k$.

\section{Asymptotic estimates}

We begin with an asymptotic calculation concerning the best value of the constant in Theorem 1. As before we put $b_{j}=a_{j}+u_{j}$ so that $k=\sum_{i=1}^{n} 1 /\left(a_{j}+u_{i}-a_{i}\right)=1 / u_{j}+\sigma_{j}+O\left(u_{j}\right)$ as $u_{j} \rightarrow 0_{+}$. Inverting this relation gives

$$
u_{j}=\frac{1}{k}+\frac{\sigma_{j}}{k^{2}}+O\left(k^{-3}\right) \text { and so } r_{j}=d_{j}+\frac{\sigma_{j+1}-\sigma_{j}}{k^{2}}+O\left(k^{-3}\right) \text { as } k \rightarrow \infty
$$

But for any $j$,

$$
\begin{aligned}
\sigma_{j+1}-\sigma_{j} & =\sum_{i \neq j+1} \frac{1}{a_{j+1}-a_{i}}-\sum_{i \neq j} \frac{1}{a_{j}-a_{i}} \\
& =\frac{2}{d_{j}}-\sum_{i<j} \frac{a_{j+1}-a_{j}}{\left(a_{j+1}-a_{i}\right)\left(a_{j}-a_{i}\right)}-\sum_{i>j+1} \frac{a_{j+1}-a_{j}}{\left(a_{i}-a_{j+1}\right)\left(a_{i}-a_{j}\right)}<\frac{2}{d_{j}}
\end{aligned}
$$

since both summations are positive. Hence taking $j=j_{0}$ so that $d_{j_{0}}=d=\delta(f)$ we have

$$
\delta\left(f^{\prime}-k f\right) \leqq r_{j_{0}}=d+\frac{2}{d k^{2}}+O\left(k^{-3}\right)
$$

as $k \rightarrow \infty$ and so the constant 12 in Theorem 1 can be replaced by $2+o(1)$ as $k \rightarrow \infty$.

To investigate the estimate in the corollary we argue as follows. Recall that for $k>0$ we have

$$
F(x)=(D-k I)^{-1} f(x)=-e^{k x} \int_{x}^{\infty} e^{-k t} f(t) d t
$$

We consider the situation in which the zeros of $f$ are equally spaced, say $f(x)=$ $\prod_{0}^{n-1}(x+i d), d>0$ and estimate how large $k$ must be to ensure that $F$ has a sign change between $x=0$ and $x=-d$. The conjecture that equal spacing gives the extremal configuration is not proved but is supported by numerical evidence, as was the case for the operator $D-k I$ in [3].

Suppose then that $f(x)=\prod_{0}^{n-1}(x+i d), d=\delta(f)>0$. Then $F(0)=-\int_{0}^{\infty} e^{-k t} f(t) d t$ is obviously negative and so we need an estimate for the size of $k$ to ensure that $F(-d)=-e^{-k d} \int_{-d}^{\infty} e^{-k t} f(t) d t$ is positive. To obtain this we consider the two integrals $I_{0}=\int_{-d}^{0} e^{-k t} f(t) d t<0$ and $I_{1}=\int_{0}^{\infty} e^{-k t} f(t) d t>0$ separately and find how large $k$ must be to make $\left|I_{0}\right|>I_{1}$.

To estimate $I_{0}$ note that for $-d \leqq x \leqq 0$ we have $|f(x)| \leqq(n-2) ! d^{n-2}|x(d+x)|$ and so 


$$
\begin{aligned}
\left|I_{0}\right| & \leqq(n-2) ! d^{n-2} \int_{-d}^{0}|t(d+t)| e^{-k t} d t \\
& =k^{-2}(n-2) ! d^{n-2}\left[d\left(e^{k d}+1\right)-2\left(e^{k d}-1\right) / k\right] \\
& \leqq 2(n-2) ! d^{n-1} e^{k d} / k^{2}
\end{aligned}
$$

after two integrations by parts.

To estimate $I_{1}$ note that for $x>0$ we have $f(x) \geqq(n-1) ! d^{n-1} x$ and so

$$
I_{1} \geqq(n-1) ! d^{n-1} \int_{0}^{\infty} t e^{-k t} d t=k^{-2}(n-1) ! d^{n-1} .
$$

Comparing these two estimates shows that values of $k$ with $2 e^{k d}<n-1$, i.e. $k<$ $\log ((n-1) / 2) / d$ will give $\left|I_{0}\right|<I_{1}$ and so will not produce the required sign change of $F$. Hence $k \geqq \log ((n-1) / 2 / d$ is necessary for $F$ to have all real zeros. Comparing this with the corollary shows that the estimates agree in order of magnitude except for the $h_{2}(n)$ term which is of order $\log \log n$.

\section{REFERENCES}

1. R. Gelca, A short proof of a result on polynomials, Amer. Math. Monthly 100 (1993), 936937.

2. P. L. WALKer, Separation of the zeros of polynomials, Amer. Math. Monthly 100 (1993), 272-273.

3. P. L. WALKER, Bounds for the separation of the zeros of polynomials. J. Australian Math. Soc., Ser.A 59 (1995), 330-342.

College of SCIEnCE

P.O. Box 36

Sultan Qaboos University

AL-KHOD, 123

Muscat

Sultanate of Oman

e-mail address: SCW0852@SQU.EDU 